\title{
Diagnóstico molecular y prevalencia de infecciones por Chlamydia trachomatis en pacientes sintomáticas y asintomáticas de una población del estado de Zulia, Venezuela
}

\author{
Nailet Arráiz R., Messaria Ginestre P., Armindo Perozo M., \\ Maribel Castellano G., Baldimiro Urdaneta y María M. García G.
}

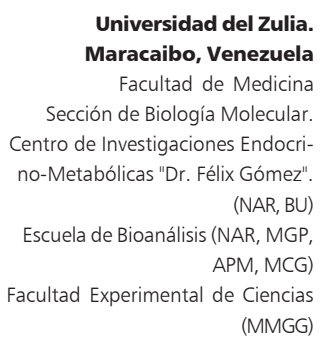

Recibido: 10 marzo 2006 Aceptado: 2 agosto 2006

Correspondencia a: Nailet Arráiz Rodríguez narraiz@cantv.net

\section{Molecular diagnosis and Chlamydia trachomatis infections prevalence in symptomatic and asymptomatic patients of a population of the Zulia State, Venezuela}

One of the world-wide most prevalent sexually transmitted pathogens is Chlamydia trachomatis. Technical limitations in their detection difficult the estimation of it true prevalence. The aim of this study was to investigate $C$. trachomatis prevalence in symptomatic and asymptomatic patients by two PCR assays. DNA extraction of endocervical swabs from 105 patients were performed and the samples tested by PCR. Positive samples for $C$. trachomatis were considered if fragments in both PCR reactions were observed. The prevalence rates were $13.7 \%, 7.4 \%$ and $10.4 \%$ for the symptomatic, asymptomatic patients and the total population, respectively. Highest prevalence was registered in symptomatic 20 to 30 years old patients. (19.2\%) Chlamydia trachomatis prevalence in the population was relatively high and is desirable to apply diagnostics strategies as the reported one in this study to detect the pathogen and offer an adequate and opportune treatment to patients.

Key words: Chlamydia trachomatis, polymerase chain reaction, endocervical swabs, symptomatic asymptomatic.

Palabras claves: Chlamydia trachomatis, reacción de polimerasa en cadena, hisopados endocervicales, sintomáticas, asintomáticas.

Este trabajo recibió co-financiamiento del Consejo de Desarrollo Científico y Humanístico de la Universidad del Zulia (Programa N ${ }^{\circ}$ CC-0241-02).

\section{Introducción}

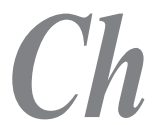

lamydia trachomatis es una bacteria gramnegativa intracelular, considerada uno de los patógenos de transmisión sexual prevalentes en el mundo ${ }^{1-5}$. Las infecciones urogenitales causadas por $C$. trachomatis cursan con múltiples manifestaciones clínicas incluyendo cervicitis, uretritis y enfermedad inflamatoria pélvica que puede conducir a abortos e infertilidad; no obstante, la infección puede ser asintomática hasta en $80 \%$ de los $\operatorname{casos}^{6-8}$.

Debido a la gravedad de estas complicaciones, diversos países han tomado acciones para reducir la prevalencia de esta infección ${ }^{2,4,5}$; sin embargo, el diseño de programas de control epidemiológico se ve obstaculizado porque no se dispone de técnicas que reúnan criterios de sensibilidad satisfactorios.

La técnica de referencia para el diagnóstico de infecciones por Chlamydia sigue siendo el cultivo, la que teniendo una especificidad de $100 \%$, carece de una buena sensibilidad ${ }^{2-5,9}$. Chlamydia requiere cultivos de líneas celulares, siendo éstos de alto costo y de difícil mantenimiento en un laboratorio de rutina, por lo cual muchos laboratorios han abandonado la práctica de investigación de Chlamydia en muestras clínicas.

El diagnóstico de infecciones por $C$. trachomatis en nuestro medio se basa fundamentalmente en las manifestaciones clínicas, estrategia que presenta muchas limitaciones en casos de una alta tasa de infecciones asintomáticas. Por tal motivo, desconocemos la estimación de la verdadera prevalencia en nuestra población.

El objetivo de este trabajo es investigar la prevalencia de $C$. trachomatis en una población de mujeres sexualmente activas, incluyendo pacientes sintomáticas y asintomáticas, a través de dos ensayos de amplificación por RPC con oligonucleótidos que han sido ampliamente utilizados a nivel internacional.

\section{Pacientes y Métodos}

Población y muestra. Las muestras endocervicales fueron obtenidas de 105 pacientes que asistieron a la 
consulta de un Ambulatorio Rural del Distrito Urdaneta del Estado Zulia, desde agosto hasta diciembre de 2005. La población incluyó 51 pacientes sintomáticas y 54 asintomáticas en edades comprendidas entre 18 y 48 años. Se definió como población sintomática a todas las pacientes que acudieron a consulta ginecológica con alguna manifestación clínica genitourinaria (descarga o flujo vaginal anormal, sangramiento post-coital, disuria, dolor abdominal, dismenorrea). La población de pacientes asintomáticas fue definida como todas las pacientes que acudían a la consulta como rutina de control ginecológico sin presentar manifestaciones clínicas. Se excluyó a las pacientes que recibieron terapia antimicrobiana en un período de tres semanas previo al estudio.

La muestra consistió en un hisopado endocervical tomado por personal médico entrenado obtenido mediante rotación del hisopo en la zona de transición escamo-columnar de la región endocervical. El hisopo fue introducido en un tubo conteniendo $1 \mathrm{ml}$ de tampón fosfato salino utilizado como medio de transporte. Las muestras fueron trasladadas al laboratorio, mantenidas a $4^{\circ} \mathrm{C}$ y procesadas dentro de los primeros 3 días de su recepción.

Extracción de ADN de la secreción endocervical. Se utilizó el procedimiento descrito previamente ${ }^{10}$ con algunas modificaciones. Se transfirió un volumen de $500 \mu \mathrm{L}$ de muestra a un tubo de $1,5 \mathrm{ml}$, se centrifugó a $14.000 \mathrm{x}$ g y el sedimento se resuspendió en $200 \mu \mathrm{L}$ de tampón de lisis $(50 \mathrm{mM}$ Tris-Hcl pH 7,5, 1\% Triton X100, $1 \mathrm{mM}$ EDTA, $250 \mu \mathrm{g} / \mathrm{ml}$ de proteinasa $\mathrm{K})$. Se incubó la muestra a $56^{\circ} \mathrm{C}$ durante 2 horas. Los lisados fueron extraídos con fenol-cloroformo y precipitados con etanol. El ADN se resuspendió en $30 \mu \mathrm{L}$ de tampón TE (10 mM Tris-HCl, pH 8, 1 mM EDTA, pH 8). Se utilizó $5 \mathrm{ml}$ de la muestra para ensayos de amplificación.

Detección de Chlamydia trachomatis por RPC. Para estos efectos se llevaron a cabo reacciones de amplificación utilizando los oligonucleótidos CTP1 y CTP2 dirigidos a secuencias de un plásmido endógeno de C. trachomatis $^{11-15}$, designado en este estudio RPCCTP. Se utilizó una segunda reacción de RPC como ensayo confirmatorio utilizando oligonucleótidos SERO 1A y SERO 2A, específicos de secuencias del gen que codifica la proteína de membrana externa PME1 (RPCPME1), utilizando condiciones previamente descritas ${ }^{12-14}$. El peso molecular de los fragmentos esperados fue de 201 y de 1.100 pares de bases para los ensayos RPCCTP y RPC PME1, respectivamente ${ }^{12,13}$. Se preparó una mezcla de reacción de un volumen final de $50 \mu \mathrm{L}$ que consistió en $5 \mu \mathrm{L}$ de tampón taq $\mathrm{ADN}$ polimerasa 10X (Promega), 1,5 mM $\mathrm{MgCl}_{2}, 200 \mu \mathrm{M}$ de cada desoxirribonucleótido (dATP, dCTP, dGTP y dTTP),
20 pmoles de cada oligonucleótido. Se utilizó $0,25 \mu \mathrm{L}$ de Taq ADN polimerasa 1U/1 (PROMEGA) y $5 \mu \mathrm{L}$ de muestra de ADN para cada reacción. El programa de amplificación de RPC-CTP consistió en 4 minutos a $94^{\circ}$ C y 35 ciclos de amplificación de 1 minuto a $95^{\circ} \mathrm{C}, 1$ minuto a $54^{\circ} \mathrm{C}, 1,5$ minutos a $72^{\circ} \mathrm{C}$ y un paso final de amplificación a $72^{\circ} \mathrm{C}$ por 10 minutos. Las muestras positivas para RPC-CTP se confirmaron por el segundo ensayo RPC-PME. Se denaturó a $95^{\circ} \mathrm{C}$ por $5^{\prime}$ y se programaron 49 ciclos de amplificación de $1 \mathrm{~min}$ a $95^{\circ}$ $\mathrm{C}, 1,5 \mathrm{~min}$ a $45^{\circ} \mathrm{C}, 1,5 \mathrm{~min}$ a $72^{\circ} \mathrm{C}$ y un paso final de extensión de 5 minutos a $72^{\circ} \mathrm{C}$. Las reacciones se llevaron a cabo en un termociclador MJ Research PTC$100^{\mathrm{TM}}$. Una muestra fue considerada positiva cuando se obtuvo amplificación de los fragmentos correspondientes para los dos ensayos. Se incluyó como control positivo ADN aislado de muestras positivas por inmunofluorescencia y por RPC. Como control negativo se utilizó agua destilada. Los productos de RPC se analizaron en geles de agarosa al 2\%, y los geles fueron teñidos con bromuro de etidio, visualizados en transiluminador ultravioleta y fotografiados con sistema de fotodocumentación DigiDoc, UVP.

El protocolo de investigación cumple los lineamientos éticos internacionales y fue aprobado por el Consejo de Desarrollo Científico y Humanístico de la Universidad del Zulia, Maracaibo, Venezuela. La participación de los pacientes en este estudio fue debidamente autorizada a través del documento de consentimiento previa información (CPI) en el cual se les informó los objetivos de la investigación y se les garantizó confidencialidad y privacidad de los resultados obtenidos.

\section{Resultados}

De los 105 hisopados endocervicales provenientes de pacientes sintomáticas y asintomáticas se detectaron secuencias específicas de C. trachomatis en 11 muestras, representando una tasa de prevalencia de $10,4 \%$ en la población total evaluada (Tabla 1). Todas las muestras positivas para el plásmido endógeno también resultaron positivas para las secuencias del gen PME1, lo cual se traduce en $100 \%$ de correlación entre ambos resultados.

Al analizar grupos individuales de acuerdo a la presencia de síntomas de infección, aunque la tasa de prevalencia fue mayor para las pacientes sintomáticas $(13,7 \%)$ que para el grupo de pacientes sin manifestaciones clínicas $(7,4 \%)$, no se encontró diferencia estadística significativa entre ambos grupos $(p>0,05)$.

La prevalencia global de infección guardó una asociación inversa con la edad, siendo mayor en el grupo 
Tabla 1. Prevalencia de infección por C. trachomatis en la población de acuerdo a la edad de las pacientes y presencia o no de síntomas

\begin{tabular}{|c|c|c|c|c|c|c|c|c|}
\hline \multirow{2}{*}{$\begin{array}{l}\text { Edad } \\
\text { (años) }\end{array}$} & \multicolumn{3}{|c|}{ Sintomáticas } & \multicolumn{3}{|c|}{ Asintomáticas } & \multicolumn{2}{|c|}{ Total } \\
\hline & $\mathbf{N}^{\circ}$ de casos & Casos $(+) s$ & Prevalencia & $N^{\circ}$ de casos & Casos (+)s & Prevalencia & Casos (+)s & PrevT \\
\hline $20-30$ & 26 & 5 & $19,2 \%$ & 22 & 2 & $9,0 \%$ & 7 & $14,5 \%^{\mathrm{a}}$ \\
\hline $31-40$ & 20 & 2 & $10 \%$ & 24 & 2 & $8,3 \%$ & 4 & $9,0 \%$ \\
\hline$>40$ & 5 & 0 & 0 & 8 & 0 & 0 & 0 & 0 \\
\hline Total & 51 & 7 & $13,7 \%^{b}$ & 54 & 4 & $7,4 \%^{b}$ & 11 & $10,4 \%$ \\
\hline \multicolumn{9}{|c|}{ +: Casos positivos para ambos ensayos de amplificación } \\
\hline \multicolumn{9}{|c|}{ PrevT: Prevalencia en la población total incluyendo pacientes sintomáticas y asintomáticas. } \\
\hline \multicolumn{9}{|l|}{ a: $p<0,05$} \\
\hline b: $p>0,05$ & & & & & & & & \\
\hline
\end{tabular}

Tabla 2. Signos y síntomas registrados en la población y casos positivos de infección por Chlamydia trachomatis detectados por doble ensayo de RPC

\begin{tabular}{|c|c|c|c|c|c|}
\hline \multirow[b]{2}{*}{ Signos y síntomas } & \multicolumn{2}{|c|}{ Pacientes } & \multicolumn{3}{|c|}{$\begin{array}{c}\text { Casos }(+) s \text { para } \\
\text { C. trachomatis }\end{array}$} \\
\hline & $\mathbf{n}$ & $\%$ & $\mathbf{n}$ & $\%^{a}$ & $\%{ }^{\mathbf{b}}$ \\
\hline Secreción endocervical mucopurulenta & 17 & 33,3 & 4 & 23,5 & 7,8 \\
\hline Disuria & 14 & 27,4 & 0 & 0 & 0 \\
\hline Cervicitis & 11 & 21,5 & 0 & 0 & 0 \\
\hline Sangramiento post-coital & 7 & 13,7 & 1 & 14,2 & 1,9 \\
\hline Enfermedad inflamatoria pélvica & 2 & 3,9 & 2 & 100 & 3,9 \\
\hline Total & 51 & 100 & 7 & 13,7 & 13,7 \\
\hline
\end{tabular}

de 20-30 años con una tasa de $14,5 \%$ que en el de 31 40 años en el que se observó una prevalencia de $9,0 \%$ $(\mathrm{p}<0,028)$.

El mayor número de casos de infección sintomática también se detectó en pacientes de 20-30 años (19,2\%) (Tabla 1). La detección de C. trachomatis en muestras endocervicales de pacientes de 31 a 40 fue menor, con tasas de prevalencia estimadas de 10,0 y $8,3 \%$ en pacientes sintomáticas y asintomáticas respectivamente. No se detectaron casos de infección por C. trachomatis en pacientes sobre 40 años de edad.

Entre las manifestaciones clínicas registradas con mayor frecuencia en esta población que asistió a consulta de ginecología (Tabla 2), se destacan secreción endocervical mucopurulenta $(33,3 \%)$, cervicitis $(21,5 \%)$ y disuria $(27,4 \%)$. C. trachomatis fue detectada en $23,5 \%$ del grupo de pacientes con secreciones mucopurulentas $(p>0,05)$ y en las dos pacientes con enfer- medad inflamatoria pélvica; sin embargo, el limitado número de casos no permite obtener información concluyente sobre la posible asociación entre infección y manifestaciones clínicas particulares.

\section{Discusión}

En este estudio se emplearon técnicas de amplificación de ácidos nucleicos recomendadas internacionalmente para el diagnóstico de $C$. trachomatis por su alta sensibilidad ${ }^{12-15}$. La técnica de referencia para el diagnóstico de infecciones por $C$. trachomatis sigue siendo el cultivo, limitada por su baja sensibilidad, que puede alcanzar valores de 50 a $80 \%$, comparada con las técnicas de amplificación de ácidos nucleicos ${ }^{13-16}$. En los últimos años se han desarrollado RPC comerciales, conservando altos porcentajes de especificidad y sensibilidad $^{16-19}$, pero es importante destacar que al prescindir del cultivo como técnica estándar, se recomienda utilizar un "ensayo de referencia expandido" que incluya técnicas de detección acompañada de otra técnica confirmatoria ${ }^{5,9,16,18}$. Siguiendo estas recomendaciones, en el presente estudio se incorporaron dos ensayos de amplificación por RPC (RPC-CTP y RPCPME1) para detectar secuencias de ADN de $C$. trachomatis en muestras clínicas y cuantificar la prevalencia de infecciones por este patógeno en una población.

La RPC-PME1 se utilizó como prueba confirmatoria de resultados obtenidos con reacciones RPC-CTP, demostrándose $100 \%$ de concordancia entre ambos ensayos, lo cual difiere de otros reportes que demuestran fallas en la amplificación del gen PME1, debiendo llevar a cabo posteriores RPC utilizando oligonucleótidos dirigidos a secuencias internas del producto de la primera reacción ${ }^{6,9,14,20,21}$. Estas diferencias podrían 
ser explicadas porque en este trabajo, las RPC se hicieron con ADN después de procedimientos de extracción y purificación, brindando mayor disponibilidad de ADN blanco, a diferencia de otros trabajos que utilizan directamente lisados de muestras clínicas y esto puede traducirse en cantidades subóptimas de templado, principalmente del gen PME1 el cual se encuentra en una sola copia/genoma ${ }^{6,9,13,20}$.

La prevalencia encontrada en el presente estudio fue similar a la reportada por otros autores, con tasas que se ubican en rangos desde 1 a $10 \%{ }^{17,21-23}$ para poblaciones de baja a mediana prevalencia y hasta 11 a $20 \% 6,9,14,21$ para poblaciones de alta prevalencia, de manera que la tasa de prevalencia de infecciones por C. trachomatis en la población analizada puede considerarse relativamente alta $(10,4 \%)$ para la población total y alta para pacientes jóvenes (14,5\%).

La mayor frecuencia en mujeres jóvenes ha sido documentada a través de diversos estudios, particularmente en mujeres menores de 20 años $^{5,7,16,19,21,22}$. En el presente estudio no participaron pacientes bajo 20 años de edad, pero el mayor número de casos detectados de infecciones por $C$. trachomatis se ubicó en el grupo etario más joven (20-30 años) y declinó en pacientes sobre 30 años de edad. La frecuencia fue mayor en el grupo de pacientes con manifestaciones clínicas caracterizadas por secreción mucopurulenta, sangramiento post-coital y enfermedad inflamatoria pélvica, sugiriendo que estos síntomas deberían considerarse para la investigación de $C$. trachomatis; $\sin$ embargo, se requiere evaluar un mayor número de muestras para validar el análisis estadístico y demostrar la posible asociación entre infección por este patógeno y manifestaciones clínicas particulares. Algunos autores señalan que la cantidad de cuerpos elementales de $C$. trachomatis se correlaciona con signos de inflamación y presencia de síntomas ${ }^{16}$.

La observación de una disminución de casos de infección por $C$. trachomatis en la población de pacientes sobre 30 años de edad, es consistente con otros hallazgos en diversas poblaciones analizadas ${ }^{7,16,19}$, lo cual ha sido atribuido a diferencias anatómicas dadas por una menor exposición del epitelio escamo-columnar de la región endocervical en mujeres mayores ${ }^{14}$, aunque también se han señalado factores demográficos adicionales, tales como número de parejas sexuales, número de partos, raza negra y bajas condiciones socioeconómicas ${ }^{8,9}$.

Aunque la mayor prevalencia fue encontrada en mujeres sintomáticas, alertarmos acerca de la necesidad de investigar mujeres asintomáticas infectadas, las cuales, por no presentar síntomas no son investigadas ni tratadas, convirtiéndose en víctimas potenciales de serias complicaciones y en fuentes de propa- gación de la infección. Adicionalmente sería muy importante llevar a cabo en el futuro estudios epidemiológicos que permitan demostrar si algunas genovariantes de nuestra población están asociadas a infecciones sintomáticas y asintomáticas.

En conclusión, la edad y posiblemente la presencia de síntomas, parecen ser factores importantes para la detección de $C$. trachomatis en la población evaluada y bajo las condiciones de este estudio.

La estrategia de diagnóstico molecular utilizada en este trabajo podría ser de gran valor como herramienta para la investigación de infecciones por C. trachomatis, para evaluar la prevalencia de este patógeno en poblaciones sintomáticas y asintomáticas a gran escala y brindar posibilidades terapéuticas acertadas y oportunas a las pacientes afectadas.

\section{Resumen}

Chlamydia trachomatis es uno de los patógenos de transmisión sexual predominantes en el mundo. Limitaciones técnicas para su diagnóstico dificultan la estimación de su verdadera prevalencia. El objetivo de este trabajo fue investigar la prevalencia de infecciones por $C$. trachomatis en pacientes sintomáticas y asintomáticas, a través de dos ensayos de amplificación por RPC. Se hizo extracción de ADN de hisopados endocervicales de 105 pacientes y se analizaron por RPC. Se consideraron positivas para C. trachomatis, aquellas muestras en las cuales se detectaron fragmentos en ambas reacciones de RPC. Las tasas de prevalencia fueron de $13,7 \%, 7,4 \%$ y $10,4 \%$ para las pacientes sintomáticas, asintomáticas y la población total, respectivamente. La mayor prevalencia se registró en pacientes sintomáticas del grupo etario de 20 a 30 años (19,2\%). La prevalencia de infecciones por $C$. trachomatis en la población fue relativamente alta y se debe resaltar la importancia de aplicar estrategias diagnósticas, como la reportada en el presente estudio, que permitan detectar el patógeno y brindar a las pacientes terapias adecuadas y oportunas.

\section{Agradecimientos}

Al Consejo de Desarrollo Científico y Humanístico de la Universidad del Zulia por el co-financiamiento de esta investigación (Programa $\mathrm{N}^{\circ}$ CC-0241-02). A la Oficina de Planificación del Sector Universitario (OPSU) por su contribución en el fortalecimiento del laboratorio de Biología Molecular del Centro de Investigaciones Endocrino-Metabólicas Dr. Félix Gómez de la Facultad de Medicina de la Universidad del Zulia. 


\section{Referencias}

1.- Adler M W. Sexually transmitted diseases control in developing countries. Genitourin Med 1996; 72: 83-8.

2.- Centers for Disease Control and Prevention. Sexually transmitted diseases treatment guidelines 2002. MMWR Morb Mortal Wkly Rep 2002; RR-51: 32-4.

3.- Yamazaki T, Hagiwara T, Kishimoto T, Sasaki N, Takahashi S, Ishihara O, et al. Distribution of Chlamydia trachomatis among female prostitutes and non prostitutes in Thailand, and non prostitutes in Japan during the mid-90s. Jpn J Infect Dis 2005; 58: 211-3.

4.- Tan H H, Chan R. Use of polimerase chain reaction on pooled cervical swabs to detect Chlamydia trachomatis infections in female sex workers in Singapore. Singapore Med J 2005; 46: 215-8.

5.- Watson E, Templeton A, Russell I, Paavonen J, Mardh P, Stary A, et al. The accuracy and efficacy of screening test for Chlamydia trachomatis: a systematic review. J Med Microbiol 2002; 51: 1021-31.

6.- Hay P E, Ghaem-Maghami S. Chlamydia and non-gonococcal urethritis. Curr Opin Infect Dis 1997; 10: 44-9.

7.- Morré S A, Rozendal L, Van Vankelgoed I G, Boeke A J, Van Voorst Vader P C. Schirm J, et al. Urogenital Chlamydia trachomatis serovars in men and women with a symptomatic or asymptomatic infection: an association with clinical manifestations. J Clin Microbiol 2000; 38: 2292-6.

8.- Cates W Jr, Wasserheit J N. Genital chlamydial infections: epidemiology and reproductive sequelae. Am J Obstet Gynecol 1991; 164: 1771-81.

9.- Black C M. Current methods of laboratory diagnosis of Chlamydia trachomatis infections. Clin Microbiol Rev 1997; 10: 160-84.
10.- Goessen W H, Kluitmans J A, Den Toom N, Van Rijsoort-Vos TH, Niesters B G, Stolz E, et al. Influence of volume of sample processed on detection of Chlamydia trachomatis in urogenital samples by PCR. J Clin Microbiol 1995; 33: 251-3.

11.- Palmer E M, Falkow S. A common plasmid of Chlamydia trachomatis. Plasmid 1986; 16: 52-62.

12. - Lan J, Jan M, Walboomers J M, Roosendaal R, van Doornum G J, MacLaren, et al. Direct detection and genotyping of Chlamydia trachomatis in cervical scrapes by using polymerase chain reaction and restriction fragment length polymorphism analysis. J Clin Microbiol 1993; 31: 1060-5.

13.- Lan J, Melgers I, Meijer CJ, Walboomers J M, Roosendaal R, Burger C, et al. Prevalence and serovar distribution of asymptomatic cervical Chlamydia trachomatis infections as determined by the highly sensitive PCR. J Clin Microbiol 1995; 33: 3194-7.

14. - Morré S A, Ossewaarde J M, Lan J, Van Doornum J M, Walboomers J M, Maclaren D M, et al. Serotyping and genotyping of genital Chlamydia trachomatis isolates reveals variants and serovars $\mathrm{Ba}, \mathrm{G}$ y $\mathrm{J}$ as confirmed by ompl nucleotide sequence analysis. J Clin Microbiol 1998; 36: 345-51.

15.- Quinn T C. Recent advances in the diagnosis of sexually transmitted diseases. Sex Transm Dis 1994; 21 (Suppl 2): S19-S27.

16.- Marrazzo J M, Johnson R, Green T A, Stamm W E, Schachter J, Bolan G, et al. Impact of patient characteristics on performance of nucleic acid amplification test and DNA probe for detection of Chlamydia trachomatis in women with genital infections. J Clin Microbiol 2005; 43: 577-84.

17.- Puolakkainen M, Hiltunen-Back E, Reunala T, Suhonen S, Lahteenmaki P, Lehtinen $\mathrm{M}$, et al. Comparison of perfor- mance of two commercially available test, a PCR assay and a ligase chain reaction test, in detection of urogenital Chlamydia trachomatis infection. J Clin Microbiol 1998; 36: 1489-93.

18.- Schepetiuk S, Tuckweng K, Martin L, Waddell R, Higgins G. Detection of Chlamydia trachomatis in urine samples by nucleic acid test: Comparison with culture and enzyme Immunoassay of genital swabs specimens. J Clin Microbiol 1997; 35: 3355-7.

19.- George J A, Panchatcharam T S, Paramasivam R, Balasubramanian S, Chakrapani V, Murugan G. Evaluation of diagnosis efficacy of PCR methods for Chlamydia trachomatis infection in genital and urine specimen of symptomatic men and women in India. Jpn J Infect Dis 2003; 56: 88-92.

20. - Lan J, Ossewaarde J M, Walboomers J M, Meijer C J, Van Den Brule A J. Improved PCR sensitivity for direct genotyping of Chlamydia trachomatis serovars by using a nested PCR. J Clin Microbiol 1994; 32: 528-30.

21.- Lauderdale T S, Landers L, Thorneycroft I, Chapin K. Comparison of the PACE 2 assay, two amplification assays, and clear view EIA for detection of Chlamydia trachomatis in females endocervical and urine specimens. J Clin Microbiol 1999; 37: 2223-9.

22.- Crotchfelt K A, Welsh L E, DeBonville D, Rosenstraus M, Quinn T. Detection of Neisseria gonorrhoeae and Chlamydia trachomatis en specimens from men and women by a coamplification PCR assay. J Clin Microbiol 1995; 35: 1536-40.

23.- Chernesky M A, Jang D, Lee H, Bruczak J D, Hu H, Sellors J, et al. Diagnosis of Chlamydia trachomatis in infections in men and women by testing first-void urine by ligase chain reaction. J Clin Microbiol 1994; 32: 2682-5. 